\title{
Catalog of $3<z<5.5$ Quasar Candidates Selected among XMM-Newton Sources and Its Spectroscopic Verification
}

\author{
Georgii Khorunzhev $^{1 *}$, Sergey Sazonov ${ }^{1}$, Rodion Burenin ${ }^{1}$ and Maxim Eselevich ${ }^{2}$ \\ ${ }^{1}$ High Energy Astrophysics, Space Research Institute, Russian Academy of Sciences, Moscow, Russia, ${ }^{2}$ Laboratory of \\ Infrared Methods in Astrophysics, Institute of Solar-Terrestrial Physics, Russian Academy of Sciences, Irkutsk, Russia
}

OPEN ACCESS

Edited by:

Mauro D'Onofrio,

Università degli Studi di Padova, Italy

Reviewed by:

Paola Severgnini,

Brera Astronomical Observatory, Italy Fabio La Franca,

Università degli Studi Roma Tre, Italy

${ }^{*}$ Correspondence:

Georgii Khorunzhev horge@iki.rssi.ru

Specialty section:

This article was submitted to Milky Way and Galaxies,

a section of the journal

Frontiers in Astronomy and Space

Sciences

Received: 31 August 2017 Accepted: 24 October 2017 Published: 13 November 2017

Citation:

Khorunzhev G, Sazonov S, Burenin R and Eselevich M (2017) Catalog of $3<$ $z<5.5$ Quasar Candidates Selected among XMM-Newton Sources and Its Spectroscopic Verification. Front. Astron. Space Sci. 4:37. doi: 10.3389/fspas.2017.00037
We have compiled a catalog of 903 quasar candidates (including known quasars) at $3<z<5.5$ selected among X-ray sources from the XMM-Newton serendipitous survey (3XMM-DR4 catalog). We used photometric SDSS, 2MASS, and WISE data to select the objects. The surface number density of objects in our sample exceeds that in the SDSS spectroscopic quasar sample at the same redshifts by a factor of 1.5. We have performed spectroscopic observations of a subsample of new quasar candidates using a new low- and medium-resolution spectrograph at the 1.6-m AZT-33IK telescope (Mondy, Russia) and demonstrated that the purity of these candidates is about $65 \%$. We have discovered one of the most distant $(z=5.08)$ X-ray selected quasars.

Keywords: active galactic nuclei, quasars, X-ray surveys, photometric redshifts, spectroscopy, XMM-Newton, SDSS

\section{INTRODUCTION}

Searching for quasars at $z>3$ is one of the key elements of studying the growth history of supermassive black holes and the evolution of massive galaxies in the Universe. For this purpose, multiwavelength observations of a large number of quasars are needed. Particularly valuable are $\mathrm{X}$-ray observations: the intensive $\mathrm{X}$-ray emission is directly connected with powerful processes of accretion onto the black hole. In order to improve our fairly poor knowledge of the evolution of the $\mathrm{X}$-ray luminosity function of quasars at $z>3$, larger X-ray samples of distant quasars are needed.

We have made an attempt to obtain a large sample of luminous X-ray quasars at $z>3$ in the fields of the serendipitous XMM-Newton 3XMM-DR4 (Watson et al., 2009) survey at Galactic latitudes $|b|>20^{\circ}$ using photometric data from SDSS (Eisenstein et al., 2011), 2MASS (Skrutskie et al., 2006) and WISE (Wright et al., 2010). The 3XMM-DR4/SDSS overlapping area is 300 sq. deg, exceeding by several times the areas covered by known samples of distant X-ray quasars such as Champ (Kalfountzou et al., 2014) and XMM-XXL (north) (Menzel et al., 2016). The sample of Kalfountzou et al. (2014) covers $\approx 33$ sq. deg and includes 87 X-ray selected optically bright (detected by SDSS) quasars. Half of them have photometric redshifts only. The spectroscopic program of (Menzel et al., 2016) has revealed $61 \mathrm{X}$-ray objects at $z_{\text {spec }}>3$ in a 18 sq. deg subfield of XMM-XXL (north). Our sample thus provides an opportunity to study a population of rare high-luminosity, distant quasars and complements recent studies of less luminous high-redshift quasars conducted using deep small-area surveys (e.g., Onoue et al., 2017; Ricci et al., 2017; Vito et al., 2017).

It should be noted that SDSS spectroscopic data provide redshift measurements for 33 thousand optically selected quasars at $z_{\text {spec }}>3$ (Alam et al., 2015) over 11,000 sq. deg in the sky. However, 
existing wide-area X-ray surveys are too shallow to study these objects. It is only the forthcoming all-sky X-ray survey by eROSITA aboard the Spektrum-Roentgen-Gamma observatory (Pavlinsky et al., 2011; Merloni et al., 2012) that will be capable of finding X-ray counterparts for a substantial fraction of known quasars at $z_{\text {spec }}>3$.

Based on broadband photometry, we obtained photometric redshift estimates $\left(z_{\text {phot }}\right)$ and compiled a catalog of 903 candidates for distant quasars with $z_{\text {phot }}>2.75$ (Khorunzhev et al., 2016). The catalog includes 515 known quasars (with spectroscopic redshifts, of which 266 are at $z_{\text {spec }}>3$ ) and 388 new quasar candidates. Most (80\%) of the spectroscopic redshifts have been obtained by SDSS (Alam et al., 2015). The remaining $z_{\text {spec }}$ have been gathered from various sources (Flesch, 2015). The new quasar candidates selected by $z_{\text {phot }}$ constitute a substantial addition to the spectroscopic sample. Thus, if most of these candidates prove to be quasars at $z>3$, the existing 3XMMDR4/SDSS sample of distant quasars will be enhanced by a factor of $\sim 1.5$.

Spectroscopic verification is necessary to determine the accuracy of our $z_{\text {phot }}$ estimates and assess the purity of our selection of quasar candidates. We have thus started a spectroscopy identification program for the new quasar candidates (Khorunzhev et al., 2017). Some initial results of this program are reported below.

\section{SAMPLE SELECTION}

K16 considered point SDSS sources at Galactic latitudes $|b|>20^{\circ}$ that have an X-ray counterpart in $3 \mathrm{XMM}-\mathrm{DR} 4^{1}$. In addition, we used near- (2MASS) and medium-infrared (WISE) photometry if available. To keep sources with reliable photometry and to get rid of M-dwarfs, we applied the following condition:

$$
\delta m_{z^{\prime}}<0.2 \& i^{\prime}-z^{\prime}<0.6,
$$

where $i^{\prime}$ and $z^{\prime}$ are the PSF magnitudes in the appropriate SDSS bands and $\delta m_{z^{\prime}}<0.2$ is the corresponding error. This is a well-known technique (e.g., Richards et al., 2002; Wu et al., 2012; Skrzypek et al., 2015) for separating M-dwarfs from distant quasars. Stars have $i^{\prime}-z^{\prime}>0.8$, while quasars have $i^{\prime}-z^{\prime}<0.4$. This color remains approximately constant up to a redshift of $\approx 5.5$, until the Ly $\alpha$ line passes from $i^{\prime}$ to $z^{\prime}$. The color of quasars then becomes $i^{\prime}-z^{\prime} \approx 2$.

We then performed a broadband energy distribution fitting using the EAZY software (Brammer et al., 2008) to obtain photometric redshift estimates. We made two fitting iterations for each object using libraries of quasar (by various authors) and star (Pickles, 1998) templates. Those objects with

$$
\chi_{\text {star }}^{2} / \chi_{q s o}^{2}>1 \& z_{\text {phot }}>2.75
$$

finally constituted our catalog of 903 candidates for distant quasars selected by photometric redshift. At the same time, our procedure missed 63 known quasars at $z_{\text {spec }}>3$ (see Figure 1).

\footnotetext{
$\overline{{ }^{1} \text { http://heasarc.gsfc.nasa.gov/W3Browse/xmm-newton/xmmssc.html }}$
}

Note that we lowered the threshold of selection from $z_{\text {phot }}=3.0$ to 2.75 to achieve reasonable selection completeness at $z \sim 3$.

The completeness of our catalog in the investigated fields relative to existing spectroscopic catalogs of quasars (SDSS DR12 Alam et al. 2015 and The Half Million Quasars Flesch 2015) with $z_{\text {spec }}>3$ is about $80 \%$. The normalized median absolute deviation of photometric redshift estimates for the spectroscopically confirmed quasars $\left(\Delta z=\left|z_{\text {spec }}-z_{\text {phot }}\right|\right)$ is $\sigma_{\Delta z /\left(1+z_{\text {spec }}\right)}=0.07$, while the catastrophic outlier fraction is $\eta=9 \%\left(\right.$ when $\left.\Delta z /\left(1+z_{\text {spec }}\right)>0.2\right)$.

\section{SPECTROSCOPIC VERIFICATION}

We have performed a quasi-random spectroscopic survey of quasar candidates from the K16 catalog at the 1.6-m AZT-33IK telescope (Kamus et al., 2002; Denisenko et al., 2009) equipped with the low- and medium-resolution ADAM spectrograph (Afanasiev et al., 2016; Burenin et al., 2016). A number of additional observations were conducted with the SCORPIO I (Afanasiev and Moiseev, 2005) spectrograph at the 6-m BTA telescope.

\subsection{Observations at the AZT-33IK Telescope}

The AZT-33IK telescope is located at the Sayan Solar Observatory of the Institute of Solar-Terrestrial Physics, the Siberian branch

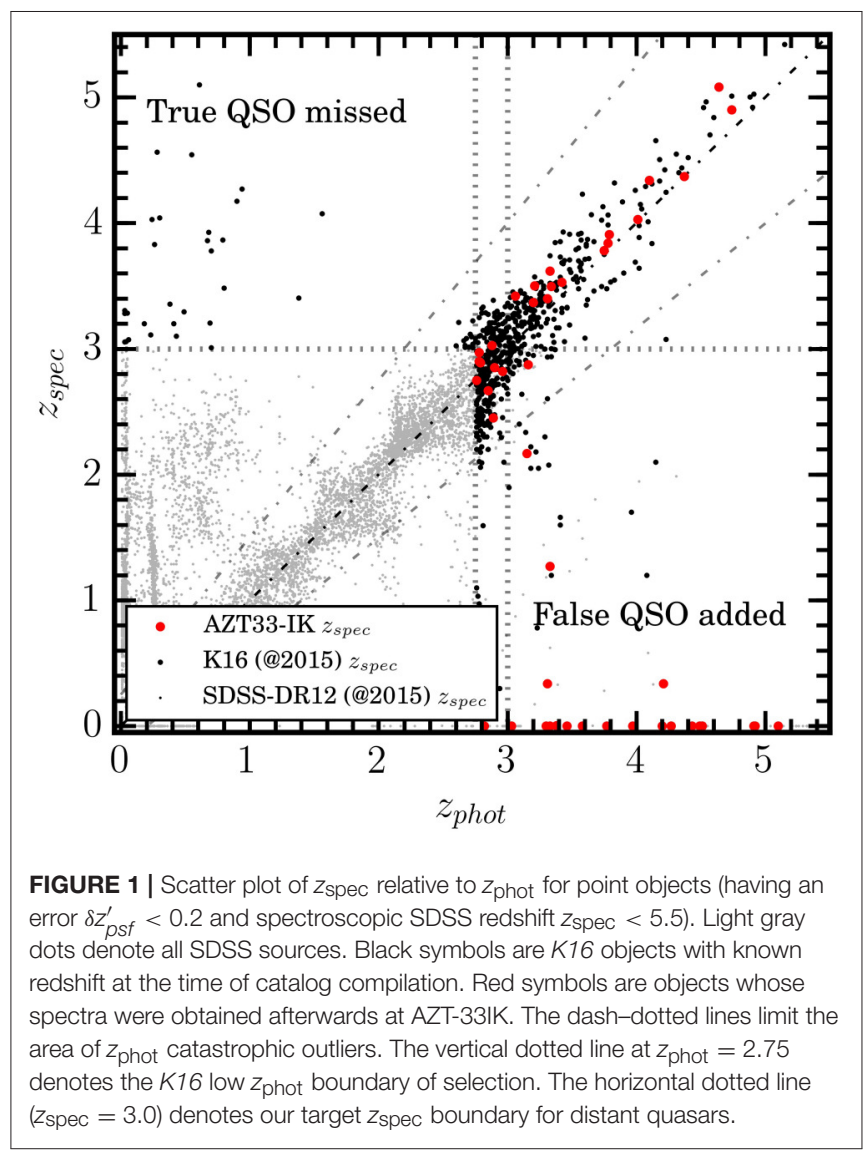


of the Russian Academy of Sciences, and has a primary mirror diameter of $1.6 \mathrm{~m}$. The low- and medium-resolution ADAM spectrograph was produced at the Special Astrophysical Observatory of the Russian Academy of Sciences and was installed on AZT-33IK in 2015. The quantum efficiency of the entire system (telescope mirror, spectrograph, grating and CCD array) reaches 50\% (Burenin et al., 2016). The ADAM spectrograph allows the spectra of objects with an apparent magnitude $\mathrm{R} \sim 19.5$ to be taken with an exposure time of half an hour. If necessary and under good weather conditions, a magnitude I $\sim 21$ can be reached with an exposure time of two hours.

By now, we have obtained the spectroscopic redshifts for 48 quasar candidates, i.e more than $10 \%$ of such objects in the K16 catalog. Approximately $60 \%$ of the observed objects proved to be quasars at $z_{\text {spec }} \gtrsim 2.5$, of which 16 have $z_{\text {spec }}>3$. We found 4 new spectroscopically confirmed quasars at $z_{\text {spec }}>4$, of which one, $3 X M M J 125329.4+305539$ is at $z_{\text {spec }}=5.08$.

\subsection{Discovery of a Quasar at $z=\mathbf{5 . 0 8}$}

3XMM J125329.4+305539 was first reported (Khorunzhev et al., 2016) as a probable quasar at $z_{\text {phot }}=4.64$ in the $K 16$ catalog, and there was no information about this source in other photometric catalogs of quasar candidates ${ }^{2}$. Its SDSS apparent magnitude is $i^{\prime} \simeq 21.0$. Its $0.5-2 \mathrm{keV}$ flux is $1.5 \times 10^{-15} \mathrm{erg} / \mathrm{s} / \mathrm{cm}^{2}$ and the corresponding X-ray luminosity is $4 \times 10^{44} \mathrm{erg} / \mathrm{s}$ (without $k$-correction).

Apart from this source, there are only 3 optically bright (i.e., with reliable SDSS photometry) X-ray quasars at $z_{\text {spec }}>$ 5.0, which are not XMM-Newton observational targets, in the $3 X M M-D R 4$ catalog. Thus, 3XMM J125329.4+305539 is one of the brightest and most distant X-ray quasars at $z_{\text {spec }}>5.0$ suitable for constructing the X-ray luminosity function at such redshifts.

\subsection{Purity and Selection Completeness}

We can estimate the purity of the K16 catalog using the obtained quasi-random spectroscopic sample. Consider the following photometric redshift intervals: $2.75 \leq z_{\text {phot }}<4,4 \leq z_{\text {phot }}<5$ and $5 \leq z_{\text {phot }}<5.5$. By purity we mean the ratio of the number of true quasars $\left(\left|z_{\text {phot }}-z_{\text {spec }}\right| /\left(1+z_{\text {spec }}\right)<0.2\right)$ to that of all objects with available spectra. Here, the value 0.2 reflects the scatter $(3$ standard deviations) of $z_{\text {phot }}$ relative to $z_{\text {spec }}$ for all of the known and spectroscopically confirmed quasars in the K16 catalog. The purity of the spectroscopic sample calculated in this way is shown in Figure 2 (circles).

For comparison, the arrows in Figure 2 indicate the lower limit on the purity of the K16 catalog estimated at the time of its compilation (before our AZT-33IK observations). This limit was deduced as the ratio of the number of true quasars with known spectroscopic redshifts and $\left|z_{\text {phot }}-z_{\text {spec }}\right| /\left(1+z_{\text {spec }}\right)<0.2$ to the total number of objects in the catalog. Recall that the new candidates without spectroscopic redshifts accounted for about $40 \%$ of the $K 16$ catalog.

${ }^{2}$ http://vizier.u-strasbg.fr

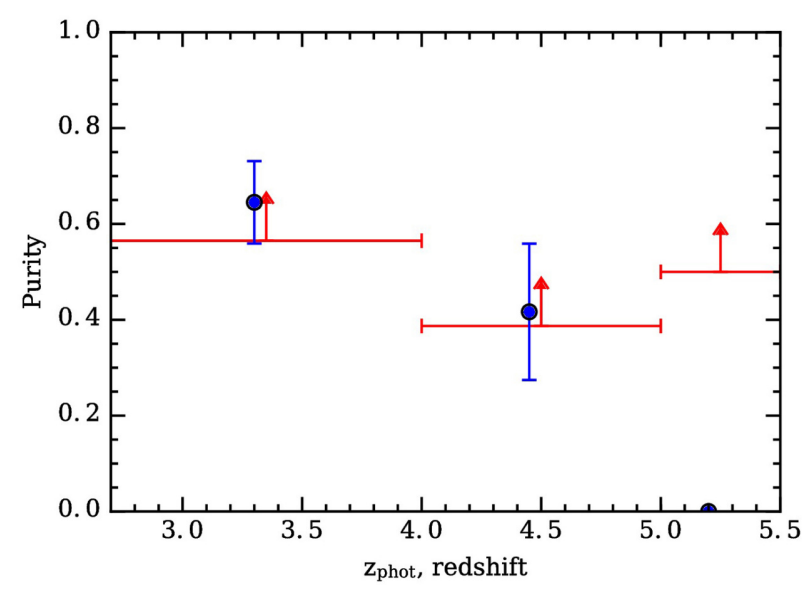

FIGURE 2 | Circles with Poissonian errorbars indicate the purity of the quasar candidates whose spectra have been taken at AZT-33IK or BTA. The arrows indicate the estimated lower limit for the purity of the K16 catalog relative to the objects with known (from literature or SDSS) spectroscopic redshifts.

Based on these preliminary results, we can expect $\sim 250$ quasars at $z \gtrsim 3$ to be firmly identified upon completion of the spectroscopy of all new quasar candidates from the $K 16$ catalog. This would significantly increase the sample of known distant $\mathrm{X}$-ray quasars.

\section{CONCLUSION}

The obtained spectra of dosens of quasars at $z \sim 3$ and especially the discovery of one of the most distant X-ray selected quasars $(3 X M M J 125329.4+305539)$ at $z_{\text {spec }}=5.08$ demonstrate that the AZT-33IK telescope of the Sayan Solar Observatory equipped with the new ADAM spectrograph is well suited for identification of distant quasars. This telescope is planned to be one of the instruments employed for optical support of the upcoming all-sky X-ray survey by the Spektrum-RoentgenGamma observatory (with its eROSITA and ART-XC telescopes, Pavlinsky et al., 2011; Merloni et al., 2012).

We have demonstrated that the existing sample of distant $\mathrm{X}$-ray quasars can be substationally increased using publicly available X-ray (XMM-Newton) and optical photometry (SDSS) data, complemented by a relatively low-cost spectroscopic identification program.

We are continuing our spectroscopic verification program for K16 candidates using the AZT-33IK and BTA telescopes and intend to obtain a complete spectroscopic sample at least for brighter X-ray sources from the K16 catalog.

\section{AUTHOR CONTRIBUTIONS}

GK provided key contributions to the compilation of the catalog, spectroscopic observations, data analysis and writing of this paper. SS supervised the research at all stages. RB contributed to the aquisition and processing of spectroscopic data. ME organized spectroscopic observations at AZT-33IK. 


\section{FUNDING}

The observations at the 6-m BTA telescope were financially supported by the Ministry of Education and Science of the Russian Federation (contract no. 14.619.21.0004, project identifier RFMEFI61914X0004).

\section{ACKNOWLEDGMENTS}

This research was partially supported by the Program of the President of the Russian Federation for support of leading scientific schools (grant NSh-10222.2016.2).

This research is based on observations obtained with XMM-Newton, an ESA science mission with instruments and

\section{REFERENCES}

Afanasiev, V. L., Dodonov, S. N., Amirkhanyan, V. R., and Moiseev, A. V. (2016). ADAM low- and medium-resolution spectrograph for 1.6-m AZT-33IK telescope. Astrophys. Bull. 71, 479-488. doi: 10.1134/S1990341316040118

Afanasiev, V. L., and Moiseev, A. V. (2005). The SCORPIO Universal Focal Reducer of the 6-m Telescope. Astron. Lett. 31, 194-204. doi: $10.1134 / 1.1883351$

Alam, S., Albareti, F. D., Allende Prieto, C., Anders, F., Anderson, S. F., Anderton, T., et al. (2015). The eleventh and twelfth data releases of the sloan digital sky survey: final data from SDSS-III. Astrophys. J. Suppl. Ser. 219:12. doi: 10.1088/0067-0049/219/1/12

Brammer, G., van Dokkum, P., and Coppi, P. (2008). EAZY: a fast, public photometric redshift code. Astrophysics 686, 1503-1513. doi: 10.1086/591786

Burenin, R. A., Amvrosov, A. L., Eselevich, M. V., Grigor'ev, V. M., Aref'ev, V. A., Vorob'ev, V.S., et al. (2016). Observational capabilities of the new medium- and low-resolution spectrograph at the 1.6-m telescope of the Sayan Observatory. Astron. Lett. 42, 295-306. doi: 10.1134/S1063773716050017

Denisenko, S. A., Kamus, S. F., Pimenov, Y. D., Tergoev, V. I., and Papushev, P. G. (2009). The AZT-33VM fast, wide-aperture telescope. J. Opt. Technol. 76, 629-631. doi: 10.1364/JOT.76.000629

Eisenstein, D. J., Weinberg, D. H., Agol, E., Aihara, H., Allende Prieto, C., Anderson, S. F., et al. (2011). SDSS-III: Massive Spectroscopic Surveys of the Distant Universe, the Milky Way, and Extra-Solar Planetary Systems. Astron. J. 142:72. doi: 10.1088/0004-6256/142/3/72

Flesch, E. W. (2015). The Half Million Quasars (HMQ) Catalogue. Publ. Astron. Soc. Aust. 32:e010. doi: 10.1017/pasa.2015.10

Kalfountzou, E., Civano, F., Elvis, M., Trichas, M., and Green, P. (2014). The largest $\mathrm{X}$-ray-selected sample of $\mathrm{z}>3$ AGNs: C-COSMOS and ChaMP. Month. Notices R. Astron. Soc. 445, 1430-1448. doi: 10.1093/mnras/stu1745

Kamus, S. F., Denisenko, S. A., Lipin, N. A., Tergoev, V. I., Papushev, P. G., Druzhinin, S. A., et al. (2002). The AZT-33VM fast, wide-aperture telescope . J. Opt. Technol. 69:674. doi: 10.1364/JOT.69.000674

Khorunzhev, G., Burenin, R., Mescheryakov, A., and Sazonov, S. (2016). Catalog of candidates for quasars at $3<\mathrm{z}<5.5$ selected among X-Ray sources from the 3XMM-DR4 survey of the XMM-Newton observatory. Astron. Lett. 42, 277-294. doi: 10.1134/S1063773716050042

Khorunzhev, G. A., Burenin, R. A., Sazonov, S. Y., Amvrosov, A. L., and Eselevich, M. V. (2017). Optical spectroscopy of candidates for quasars at $3<\mathrm{z}<5.5$ from the XMM-newton X-ray survey. A distant X-ray quasar at $\mathrm{z}=5.08$. Astron. Lett. 43, 135-145. doi: 10.1134/S1063773717030045

Menzel, M.-L., Merloni, A., Georgakakis, A., Salvato, M., Aubourg, E., Brandt, W. N., et al. (2016). A spectroscopic survey of X-ray-selected AGNs in the northern XMM-XXL field. Month. Notices R. Astron. Soc. 457, 110-132. doi: $10.1093 / \mathrm{mnras} / \mathrm{stv} 2749$

Merloni, A., Predehl, P., Becker, W., Böhringer, H., Boller, T., Brunner, H., et al. (2012). eROSITA Science Book: Mapping the Structure of the Energetic Universe. ArXiv e-prints.

Onoue, M., Kashikawa, N., Willott, C. J., Hibon, P., Im, M., Furusawa, H., et al. (2017). Minor Contribution of Quasars to Ionizing Photon Budget at $\mathrm{z} \sim 6$ : contributions directly funded by ESA Member States and NASA. Also, we used SDSS-III, 2MASS, WISE data. Funding for SDSS-III has been provided by the Alfred P. Sloan Foundation, the Participating Institutions, the National Science Foundation, and the U.S. Department of Energy Office of Science. The Two Micron All Sky Survey is a joint project of the University of Massachusetts and the Infrared Processing and Analysis Center/California Institute of Technology, funded by the National Aeronautics and Space Administration and the National Science Foundation. The Wide-field Infrared Survey Explorer is a joint project of the University of California, Los Angeles, and the Jet Propulsion Laboratory/California Institute of Technology, funded by the National Aeronautics and Space Administration.

Update on Quasar Luminosity Function at the Faint End with Subaru/SuprimeCam. Astrophys. J. Lett. 847:L15. doi: 10.3847/2041-8213/aa8cc6

Pavlinsky, M., Akimov, V., Levin, V., Lapshov, I., Tkachenko, A., Semena, N., et al. (2011). "The ART-XC instrument on board the SRG mission," in Society of Photo-Optical Instrumentation Engineers (SPIE) Conference Series, Vol. 8147 of Proc. SPIE (San Diego, CA), 814706.

Pickles, A. J. (1998). A Stellar Spectral Flux Library: 1150-25000 Å. PASP 110, 863-878.

Ricci, F., Marchesi, S., Shankar, F., La Franca, F., and Civano, F. (2017). Constraining the UV emissivity of AGN throughout cosmic time via X-ray surveys. Month. Notices R. Astron. Soc. 465, 1915-1925. doi: 10.1093/mnras/stw2909

Richards, G. T., Fan, X., Newberg, H. J., Strauss, M. A., Vanden Berk, D. E., et al. (2002). Spectroscopic target selection in the sloan digital sky survey: the quasar sample. Astron. J. 123, 2945-2975. doi: 10.1086/340187

Skrutskie, M. F., Cutri, R. M., Stiening, R., Weinberg, M. D., Schneider, S., Carpenter, J. M., et al. (2006). The two micron all sky survey (2MASS). 131, 1163-1183. doi: 10.1086/498708

Skrzypek, N., Warren, S. J., Faherty, J. K., Mortlock, D. J., Burgasser, A. J., and Hewett, P. C. (2015). Photometric brown-dwarf classification. I. A method to identify and accurately classify large samples of brown dwarfs without spectroscopy. Astron. Astrophys. 574:A78. doi: 10.1051/0004-6361/201 424570

Vito, F., Brandt, W. N., Yang, G., Gilli, R., Luo, B., Vignali, C., et al. (2017). Highredshift AGN in the Chandra Deep Fields: the obscured fraction and space density of the sub-L_* population. ArXiv e-prints.

Watson, M. G., Schröder, A. C., Fyfe, D., Page, C. G., Lamer, G., Mateos, S., et al. (2009). The XMM-Newton serendipitous survey. V. The Second XMMNewton serendipitous source catalogue. Astron. Astrophys. 493, 339-373. doi: 10.1051/0004-6361:200810534

Wright, E. L., Eisenhardt, P. R. M., Mainzer, A. K., Ressler, M. E., Cutri, R. M., Jarrett, T., et al. (2010). The wide-field infrared survey explorer (WISE): mission description and initial on-orbit performance. 140, 1868-1881. doi: 10.1088/0004-6256/140/6/1868

Wu, X.-B., Hao, G., Jia, Z., Zhang, Y., and Peng, N. (2012). SDSS Quasars in the WISE Preliminary Data Release and Quasar Candidate Selection with Optical/Infrared Colors. Astron. J. 144:49. doi: 10.1088/0004-6256/ $144 / 2 / 49$

Conflict of Interest Statement: The authors declare that the research was conducted in the absence of any commercial or financial relationships that could be construed as a potential conflict of interest.

Copyright (c) 2017 Khorunzhev, Sazonov, Burenin and Eselevich. This is an openaccess article distributed under the terms of the Creative Commons Attribution License (CC BY). The use, distribution or reproduction in other forums is permitted, provided the original author(s) or licensor are credited and that the original publication in this journal is cited, in accordance with accepted academic practice. No use, distribution or reproduction is permitted which does not comply with these terms. 